Mappemonde

Revue trimestrielle sur l'image géographique et les

formes du territoire

$130 \mid 2021$

Varia

\title{
Maillage communal et population du Brésil de 1872 à 2020
}

Hervé Théry

\section{OpenEdition}

\section{Journals}

Édition électronique

URL : https://journals.openedition.org/mappemonde/5061

DOI : 10.4000/mappemonde.5061

ISSN : 1769-7298

\section{Éditeur}

UMR ESPACE

\section{Référence électronique}

Hervé Théry, « Maillage communal et population du Brésil de 1872 à 2020 », Mappemonde [En ligne] 130 | 2021, mis en ligne le 15 mars 2021, consulté le 22 mai 2021. URL : http://

journals.openedition.org/mappemonde/5061 ; DOI : https://doi.org/10.4000/mappemonde.5061

Ce document a été généré automatiquement le 22 mai 2021.

\section{(c) (†) (2)}

La revue Mappemonde est mise à disposition selon les termes de la Licence Creative Commons Attribution - Pas d'Utilisation Commerciale - Partage dans les Mêmes Conditions 4.0 International. 


\section{Maillage communal et population du Brésil de 1872 à 2020}

\section{Hervé Théry}

Parmi les multiples ressources offertes par l'IBGE (Institut Brésilien de Géographie Statistique), utiles à qui s'intéresse aux disparités et dynamiques du territoire du Brésil, figure une publication sur l'évolution du maillage communal et de la population du pays de 1872 à 2010 . Originellement publiée sur papier en 2011, elle a ensuite été mise à disposition du public, en ligne, sous forme électronique (en PDF), et surtout sous forme de données numériques (tableau Excel du nombre d'habitants par commune et maille communale, en format shp) qui permettent de produire des cartes détaillées pour chacun des recensements et des évolutions intercensitaires.

\section{La publication}

2 La publication, est intitulée Evolução da divisão territorial do Brasil 1872-2010, c'est le numéro 17 de la série Documentos para Disseminação, Memória Institucional 17, elle a été lancée par l'IBGE 2011. Elle est donc disponible sur papier (figure 1, ISBN 978-85-240-4208-9), sur CD-ROM (ISBN 978-85-240-4209-6) et en ligne en format PDF

(https://biblioteca.ibge.gov.br/index.php/biblioteca-catalogo? view $=$ detalhes\&id=284481). 
Figure 1. Couverture de la publication

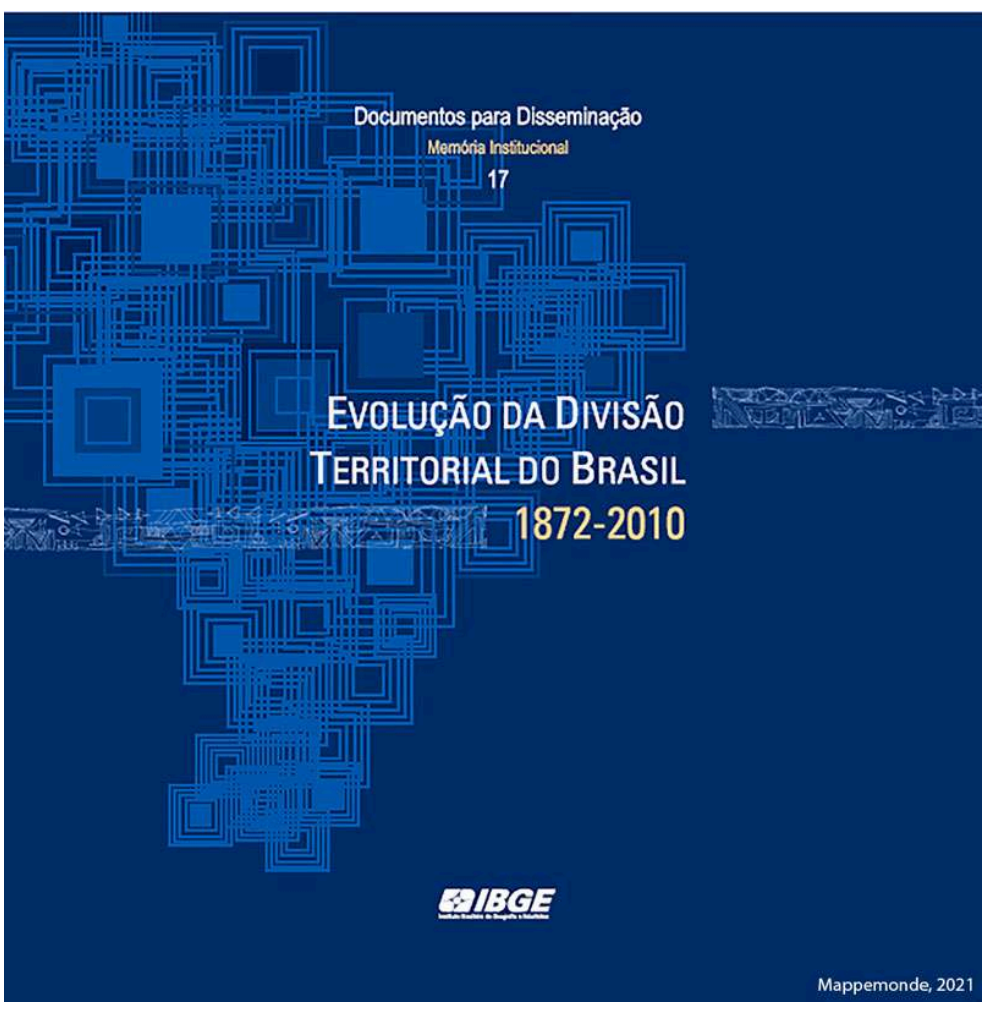

3 Dans sa présentation, Wadih João Scandar Neto, alors directeur des géosciences, indique :

«Avec la publication Evolução da divisão territorial do Brasil 1872-2010, l'Institut brésilien de géographie et de statistique - IBGE met à disposition des informations fondamentales sur la géographie et l'histoire du Brésil au cours de la période 1872 - l'année du premier recensement qui a couvert l'ensemble du territoire du pays à 2010, date du dernier recensement démographique. Les changements dans les limites des États et des communes témoignent des nombreux changements qui ont eu lieu sur le territoire, montrant comment il a été subdivisé, reconfigurant continuellement la carte politique du pays ».

4 L'introduction explique que la publication intègre des informations sur le processus de division du territoire en communes et États, combiné avec l'évolution de la population brésilienne. Il contient (au format shp) les mailles territoriales de ces deux échelles depuis le premier recensement, effectué à l'époque impériale, en 1872, et tout au long du XXe siècle, en 1900, 1911, 1920, 1933, 1940, 1950, 1960, 1970, 1980, 1991, 2000 et 2010.

5 Elle inclut aussi une séquence de cartes thématiques de l'évolution de la population totale pour chaque recensement. L'évolution de la répartition spatiale de la population sur le territoire brésilien témoigne de la force du processus d'«intériorisation » (de marche vers l'ouest) et de formation du réseau urbain, directement et indirectement associé à la croissance démographique et à celle des activités économiques. Elle a été très active, puisqu'alors que le pays ne comptait que 635 communes en 1872, il en avait un peu plus de 1500 en 1940, plus de 2500 en 1960, et plus de 5500 en $2010^{1}$.

6 Deux très longs tableaux sont insérés dans cette publication, ils en occupent la plus grande partie (de la page 60 à la page 259 sur 261), mais sont heureusement aussi disponibles en format Excel en téléchargement (voir ci-dessous). Le premier retrace la généalogie des communes brésiliennes, révélant non seulement les démembrements 
qui se sont produits au fil du temps, mais aussi les changements de toponymie et d'orthographe, tandis que le second tableau donne les chiffres de population par commune aux années de recensement.

7 Les notes techniques, précises et détaillées, indiquent que l'élaboration de «ce travail impliquait des choix méthodologiques et opérationnels qui sont explicités afin que le public soit conscient des problèmes et des restrictions rencontrés lors des travaux de compilation, de comparaison et de cartographie de la division par communes et États de 1872 à $2010 »$.

Les mailles municipales sélectionnées sont celles qui étaient en vigueur dans les années au cours desquelles des recensements ont été effectués, afin de fournir une liste des communes existant alors dans chacune des provinces ou États, ainsi que la taille de leur population. Bien qu'il n'y ait pas eu de recensements en 1910 et 1930, il a été décidé d'inclure les divisions administratives de 1911 et 1931, afin de préserver la périodicité décennale de la série.

Pour les années 1872, 1900 et 1920, le recensement donne la valeur totale de la population et à partir de 1940, lorsque l'IBGE a commencé à collecter les statistiques officielles du pays, on distingue la population totale, urbaine et rurale.

La reconstitution - ou plutôt en l'occurrence la déconstruction - de la maille municipale a été réalisée sur la base la plus récente subdivision. Ces informations ont été obtenues par consultation de la partie historique de la page Cidades@, disponible sur le site de l'IBGE (https://cidades.ibge.gov.br/), ainsi que par une recherche dans l'Enciclopédia dos municípios brasileiros (Encyclopédie des communes brésiliennes), produite par l'institution à la fin de la décennie 1950, et qui a été rééditée plus tard électroniquement.

11 La publication comprend aussi un certain nombre de cartes thématiques réalisées à partir des données rassemblées pour l'occasion. Montrant l'évolution des communes et des États, elles ont été construites sur la base de la maille de 1991, car entre celle-ci et les plus récentes il n'existe que peu de divergences, principalement dans la région du Nordeste, et résultant d'améliorations cartographiques.

Ces cartes ne sont malheureusement pas très satisfaisantes, car la culture dominante à l'IBGE étant celle de la cartographie systématique (du type de celle de l'IGN), il est pratiquement obligatoire d'y faire figurer, même pour des cartes thématiques du Brésil entier des éléments qui la surchargent inutilement, comme la grille complète des méridiens et des parallèles, les limites détaillées des communes, l'hydrographie et une abondante toponymie. Il arrive même que des valeurs absolues soient représentées par des plages de couleur, comme sur la figure $2^{2}$, et prolonger la carte par la silhouette des pays voisins est un louable souci pour ne pas tomber dans le "syndrome de l'île flottante ", mais cela charge encore la carte, qui est, au final, peu lisible. Heureusement, la mise à la disposition des lecteurs des mailles cartographiques et des données démographiques permet de construire des cartes plus claires, comme on le verra plus loin. 
Figure 2. Une des cartes, peu lisibles, de la publication

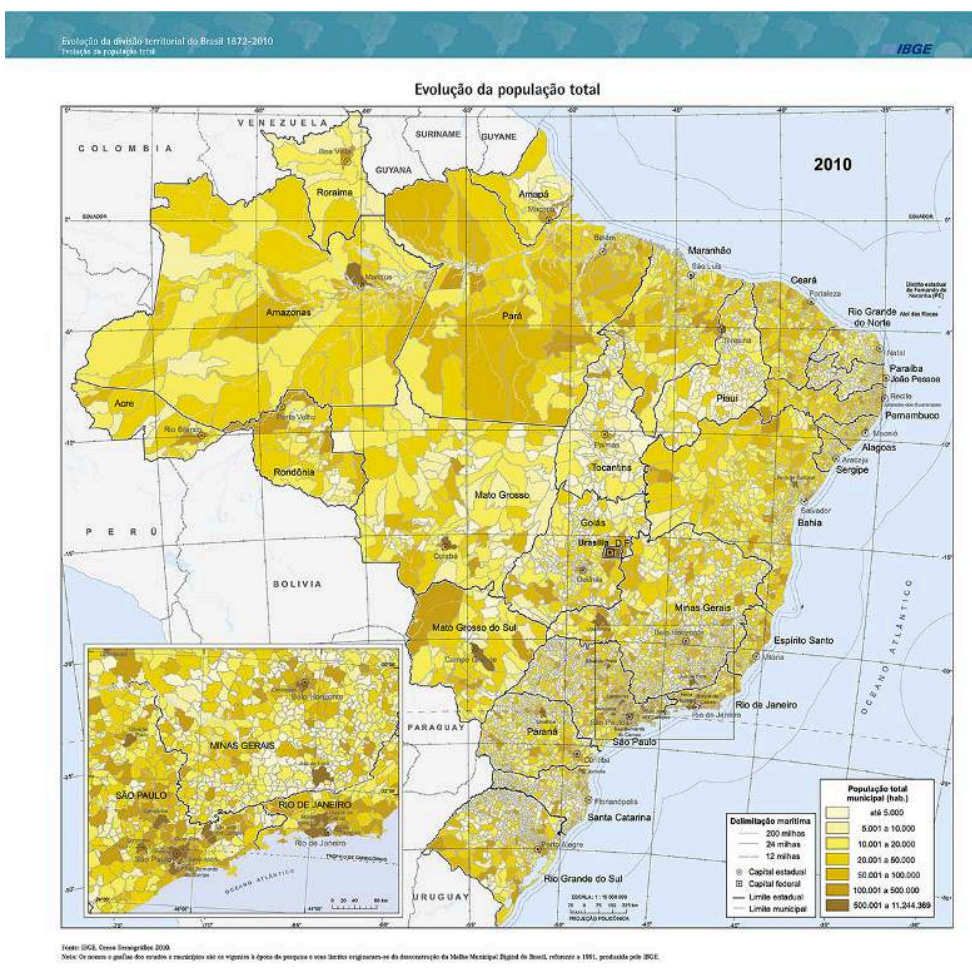

\section{Mailles et données}

Outre la mise à disposition de statistiques et de publications, le site de l'IBGE comporte une vaste section de données à télécharger. Du côté des statistiques, mais aussi du côté des géosciences (figure 3), il est possible de télécharger des cartes, des images aériennes et orbitales, des maillages, ainsi que des atlas et des fichiers Google Earth, entre autres. 
Figure 3. Le site de téléchargements de l'IBGE

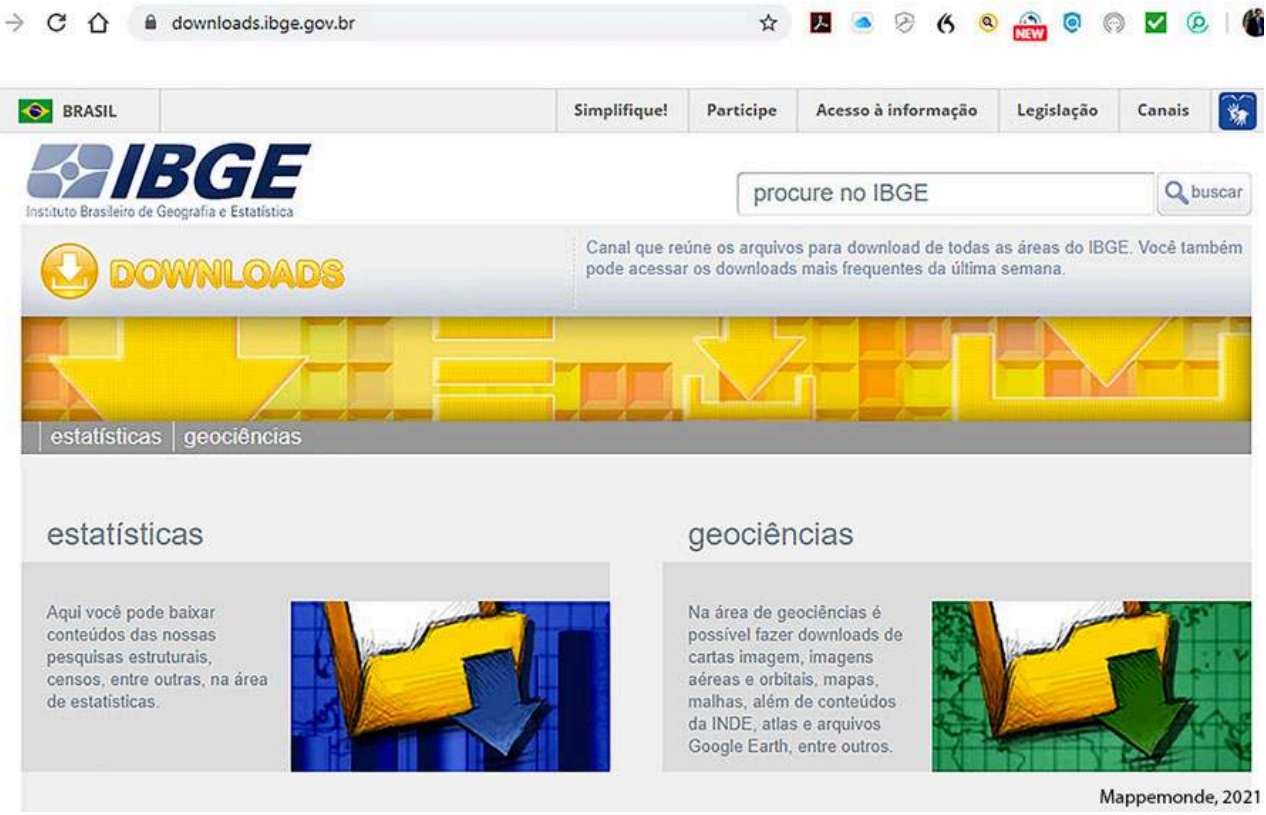

C'est dans cette section que l'on trouve les mailles municipales correspondant à chaque recensement. Il faut, pour cela, ouvrir successivement les dossiers emboités « division territoriale ", "évolution de la division territoriale du Brésil», "évolution de la division territoriale du Brésil de 1872 à 2010 », puis "communes (municípios) 1872 1991 » (figure 4). On a alors accès, pour chaque recensement, à des dossiers «zippés » qui contiennent des fichiers shp de la maille municipale (et leurs fichiers associés, dbf, prj, etc.), de celle des provinces (sous l'Empire) ou des États (après l'instauration de la République), de localisation du chef-lieu de la commune, de la province ou de l'État et de la capitale du pays. 
Figure 4. Chemin d'accès aux mailles municipales

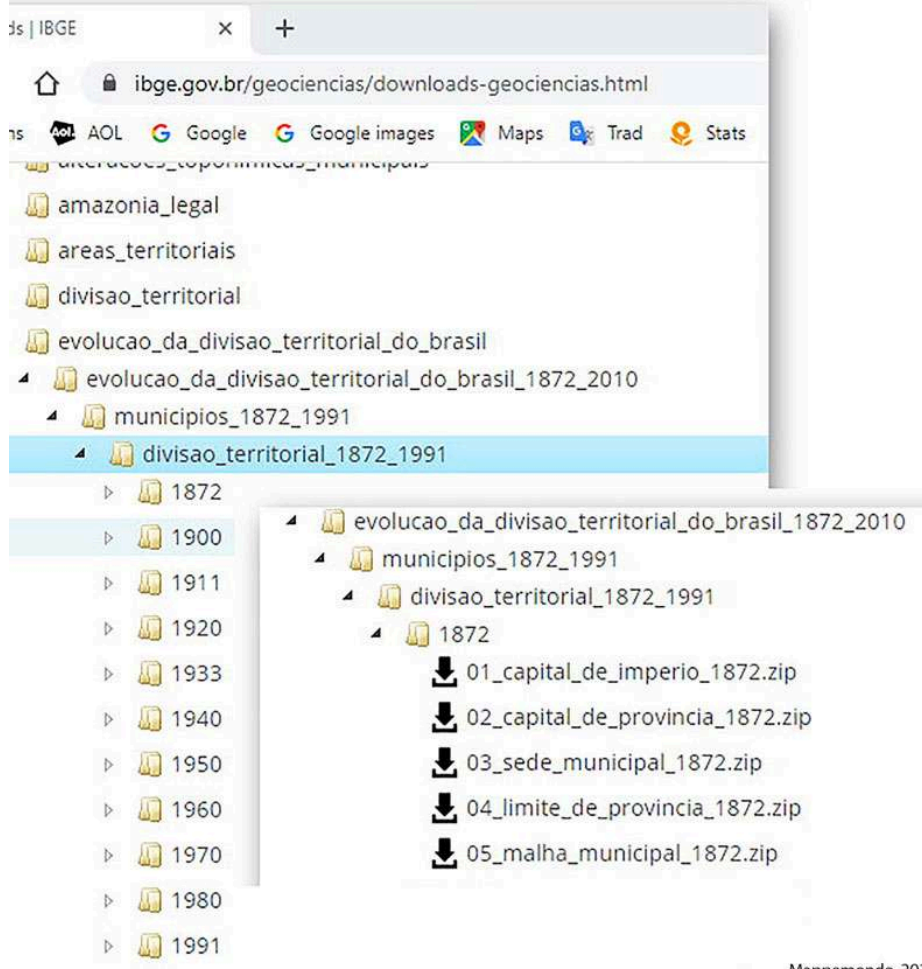

En complément de ces mailles figure aussi dans cette section de téléchargements un fichier Excel de la population de chacune des communes à chacun des recensements qui reprend le tableau de la publication (figure 5). Il comprend autant de lignes qu'il existe actuellement de communes, sur chacune d'entre elles figure sa population à ce recensement et à tous les précédents, si elle existait à l'époque, ou si ce n'est pas le cas, de la commune dont elle a été démembrée, ainsi que l'éventuel changement de nom ou de graphie du toponyme. Pour chaque étape est précisé le code IBGE qui permet de le rattacher à la maille de l'époque et donc de cartographier ces données. C'est là un travail remarquable, très minutieux et très précieux, qui permet de construire toutes sortes de cartes pour chaque époque, et de leur évolution de n'importe quelle date à n'importe quelle autre.

Figure 5. Le tableau des populations communales à chaque recensement

\begin{tabular}{|c|c|c|c|c|c|c|c|c|c|c|c|}
\hline 1 & $\stackrel{8}{8}$ & 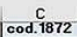 & Município 1872 & $\frac{E}{\text { pop.total }}$ & $\frac{F}{\text { pop.live }}$ & $\frac{G}{\text { pop.esorava }}$ & \begin{tabular}{|c|}
$\stackrel{A Q}{A Q}$ \\
cod.1991
\end{tabular} & $\begin{array}{l}\text { AR } \\
\text { Município } 1991\end{array}$ & $\begin{array}{c}\text { AS } \\
\text { pop.tot.1991 }\end{array}$ & $\begin{array}{c}\text { AT } \\
\text { pop.urb. } 1991\end{array}$ & AU 1 pop.rur.1991 \\
\hline 2 & 3550308 SăoPaulo & 3550308 & SåoPaulo & 31385 & 27557 & 3828 & 3550308 & Sz̊opaulo & 9646185 & 9412894 & $4 \quad 233291$ \\
\hline 3 & 3304557 Rio de Janeiro & 3099901 & Município Neutro & 274972 & 226033 & 48939 & 3304557 & Rio de Janeito & 5480768 & 5480768 & \\
\hline 4 & 2927408 Solvador & 2927408 & SžoSolvador & 129109 & 112641 & 16468 & 2927408 & Soluador & 2075273 & 2073510 & 17 \\
\hline 5 & 5300108 Brasilia & & & & & & 5300108 & Brassiia & 1601094 & 1515889 & 8520 \\
\hline 6 & 2304400 Fortaleza & 2304400 & Fortaleza & 42458 & 40524 & 1934 & 2304400 & Fortaleza & 1768637 & 1768637 & \\
\hline 7 & 3106200 BeloHorizonte & & desmembrado de Sabará & & & & 3106200 & Belo Horizonte & 2020161 & 2013257 & 69 \\
\hline 8 & 1302603 Manaus & 1302603 & Manáos & 23334 & 28706 & 628 & 1302603 & Mansus & 1011501 & 1008585 & 491 \\
\hline 9 & 4106902 Curiviba & 4106902 & Curitiba & 12651 & 11730 & & 4106902 & Cunitiba & 1315035 & 1315035 & \\
\hline 10 & 2811606 Recile & 2611606 & Recife & 116871 & 101535 & 15136 & 28111006 & Recife & 1298229 & 1298229 & \\
\hline$\pi$ & 4314902 Porto Alegre & 4314902 & Porro Alegre & 43998 & 35843 & 8155 & 4314902 & Porto Alegre & 1263403 & 1247529 & \\
\hline 12 & 1501402 Belém & 1501402 & Bechlém & 61997 & 53150 & 8847 & & Belém & 1244689 & 849187 & 39556 \\
\hline & 5208707 Goiŝnia & & & & & & 5208707 & Goiŝnia & 922222 & 913485 & \\
\hline 14 & 3518800 Guarulhos & & desmembrado de Sð̃o Paulo & & & & 3518800 & Guarulhos & 787866 & 777921 & \\
\hline 15 & 3509502 Campinos & 3509502 & Campinas & 31397 & 17712 & 13685 & 3509502 & Campinas & 847595 & 824924 & 228 \\
\hline 16 & 2111300 S5zoLuis & 2111300 & Sãoluiz & 31604 & 24578 & 7028 & 2111300 & SãoLuis & 696371 & 246244 & \\
\hline
\end{tabular}

Extrait. 


\section{Images}

En utilisant ces mailles et ces données numériques, on peut donc élaborer des cartes, différentes de celles qui figurent dans la publication, que ce soit celle des mailles ellesmêmes, de la population à chaque recensement ou de l'évolution intercensitaire de celle-ci.

\section{Mailles}

La comparaison des cartes représentant les mailles communales à la première et à la dernière date de la série est déjà instructive : sans rien savoir du nombre des habitants du pays, on pressentirait déjà qu'il a dû augmenter considérablement en observant la multiplication du nombre des communes et la réduction de leur taille (en particulier à proximité du littoral), car elles se sont constituées par démembrement des unités existant auparavant, à mesure que la population augmentait (figure 6).

Figure 6. Les mailles communales et des Provinces ou États en 1872 et 1991

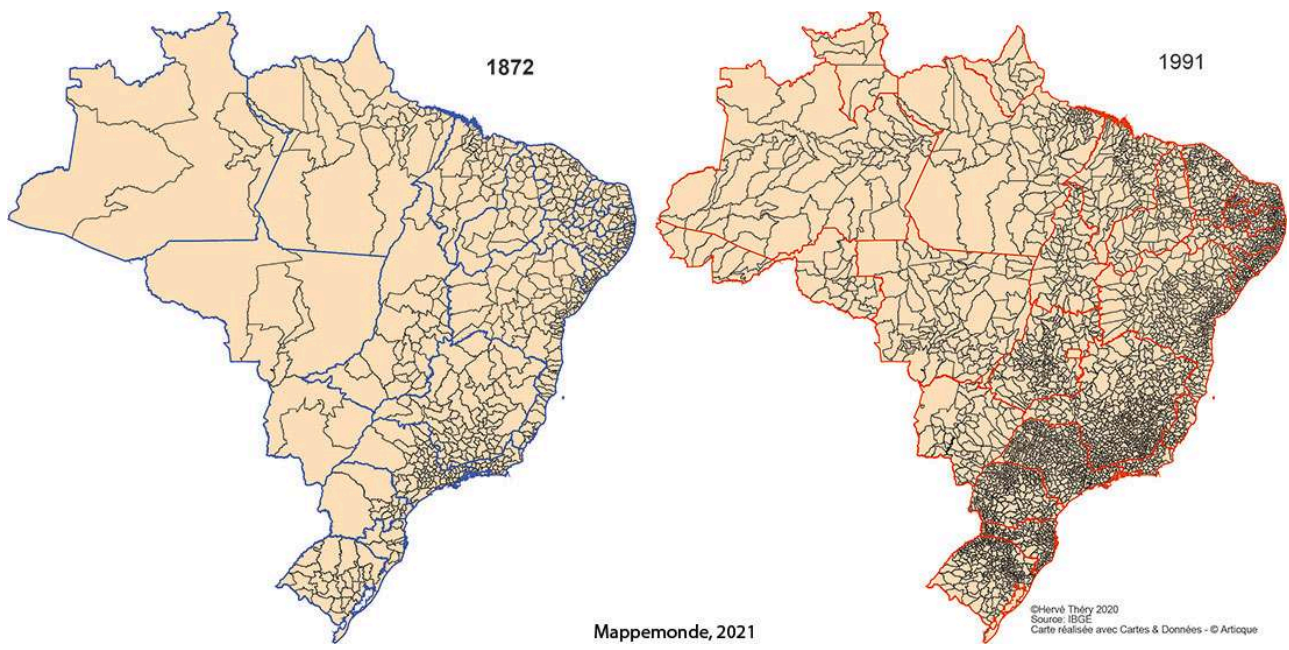

Dans sa présentation, Wadih João Scandar Neto soulignait déjà la spécificité du recensement de 1872 :

«En associant ces mailles territoriales, dans le cas du recensement de 1872, à celle de la population esclave, l'IBGE confirme son engagement à valoriser la vaste et précieuse collection de documents statistiques qui sont réunis dans cette institution »

L'esclavage n'a, en effet, été aboli au Brésil qu'en 1888, et ce recensement de 1872 détaillait donc la situation des Brésiliens qui étaient encore sous ce statut, encore légal à l'époque. Outre son évidente valeur pour qui étudie les racines culturelles de la société brésilienne, c'est un exemple de la vaste collection d'informations sur le Brésil, sa société, son économie et son territoire qui sont mis à la disposition des chercheurs, dans ses publications ou via Internet, par l'IBGE.

Ce sont donc des données précieuses, tout comme le sont les photographies qui ont pu être prises des derniers esclaves ${ }^{3}$, puisqu'avant que l'esclavage ne soit aboli au Brésil la photographie avait déjà été inventée, et pratiquée par les pionniers de cette technique ${ }^{4}$, dont l'Empereur Pedro II lui-même. 
Grâce à ces données, on a pu construire la carte de la figure 7, qui montre la distribution de la population esclave en 1872, la taille du triangle en indiquant le nombre absolu dans chaque commune du pays, et la couleur en indiquant la proportion dans la population totale. On remarque que, contrairement à ce qui est le plus souvent indiqué, cette proportion n'est pas à son plus haut dans le Nordeste producteur de sucre, mais plutôt dans les provinces centrales, Rio de Janeiro et São Paulo, car le développement des plantations de café avait permis à leurs propriétaires d'acheter des esclaves aux planteurs de canne à sucre du Nordeste, alors en difficulté. On remarque aussi les concentrations d'esclaves plus au nord, dans le Maranhão, et plus au sud, dans le Rio Grande do Sul, où ils étaient employés à la production de viande sèche dans les charqueadas. Cela contredit l'idée préconçue selon laquelle le Sud avait principalement une population blanche grâce à l'immigration européenne.

Figure 7. Nombre et proportion des esclaves dans la population en 1872

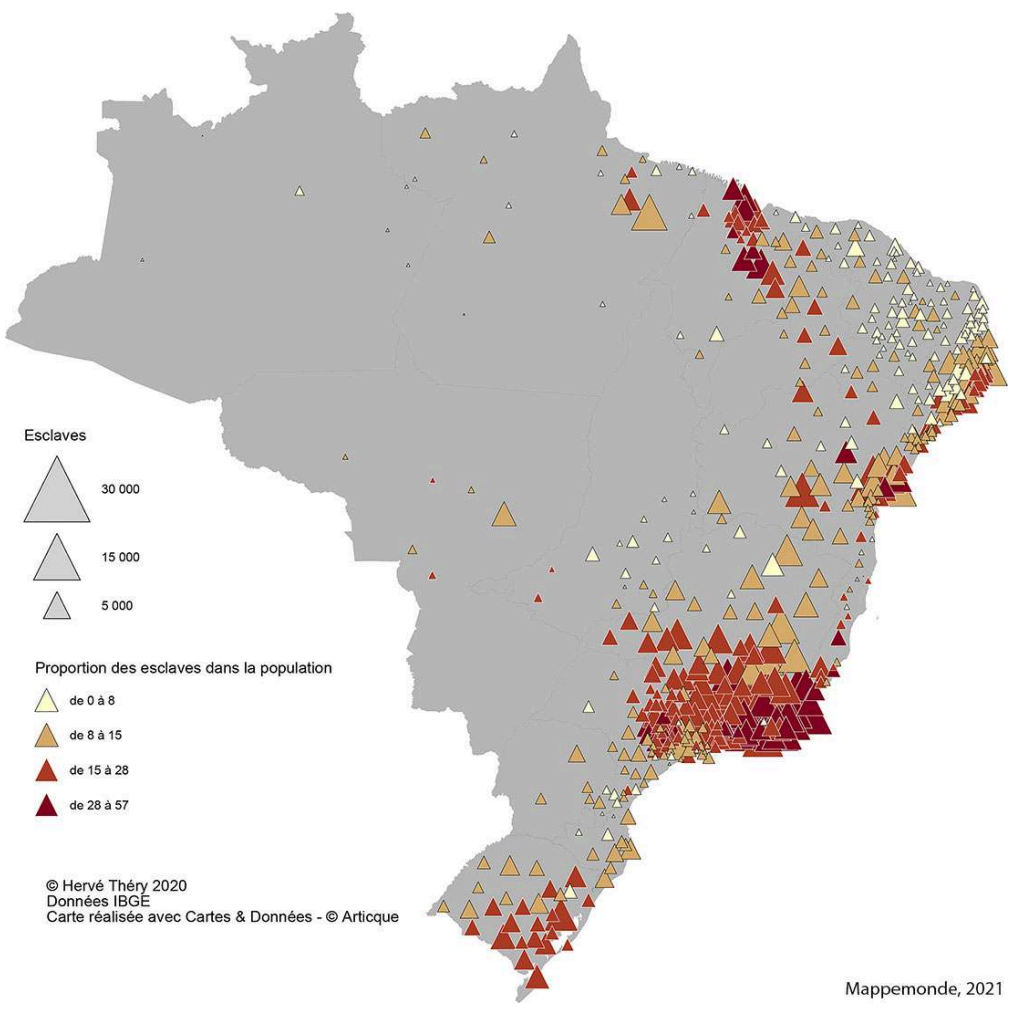

Outre ces images statiques, le fait que les données des recensements aient été rassemblées dans le même tableau, avec les mêmes codes, permet de mesurer l'évolution intercensitaire de la population.

\section{$1872-2010$}

Mesurer cette évolution à l'échelle détaillée des communes est d'autant plus intéressant que la population du pays a beaucoup augmenté depuis la fin du XIX ${ }^{e}$ siècle : alors qu'il comptait moins de 10 millions d'habitants en 1872, il en avait plus de 190 millions au recensement de 2010, et plus de 210 millions actuellement : avec 200 millions d'habitants de plus, le nombre de ses habitants a été multiplié par 21 (ou pour être plus précis, a augmenté de $2016 \%$ ) en 148 ans 
Tableau 1. Population du Brésil aux dates des recensements

\begin{tabular}{|l|l|}
\hline Recensement & Population \\
\hline 1872 & 9930478 \\
\hline 1890 & 14333915 \\
\hline 1900 & 17438434 \\
\hline 1920 & 30635605 \\
\hline 1940 & 41236315 \\
\hline 1950 & 51944397 \\
\hline 1960 & 70992343 \\
\hline 1970 & 94508583 \\
\hline 1980 & 121150573 \\
\hline 1991 & 146917459 \\
\hline 2000 & 169590693 \\
\hline 2010 & 190755799 \\
\hline $2019^{*}$ & 210.147 .125 \\
\hline
\end{tabular}

*Estimation officielle.

Source : IBGE Recensements démographiques

24 Cette croissance extrêmement rapide permet de construire des images spectaculaires, graphiques (figure 8) ou carte (figure 9) qui détaillent sa répartition par État pour le graphique, et par microrégions ${ }^{5}$ pour la carte. 
Figure 8. Graphique de la croissance de la population de 1872 à 2010
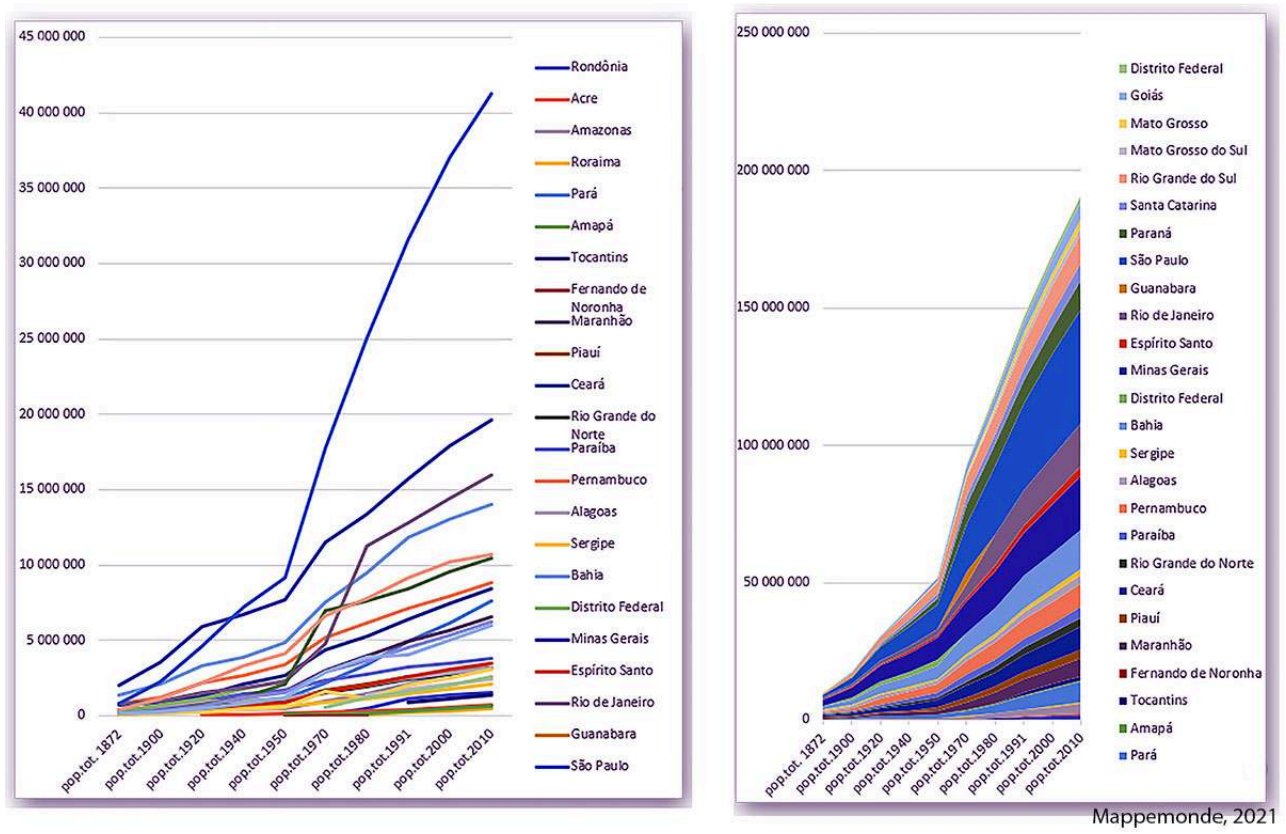

En 1872, rares étaient celles des vingt Provinces de l'Empire qui comptaient plus de 50 000 habitants. Minas Gerais et Bahia étaient les plus peuplées, suivies de Pernambuco, Ceará, São Paulo, Rio de Janeiro et Rio Grande do Sul. En 1890, grâce au boom du caoutchouc, le niveau de 50000 habitants était atteint par l'État ${ }^{6} \mathrm{~d}^{\prime}$ Amazonas.

En 1920, le Brésil ayant déjà ses frontières actuelles après l'incorporation de l'Acre et d'autres rectifications des limites extérieures, les 22 États et le District fédéral (à l'époque la ville de Rio de Janeiro et ses environs) comptaient une population de plus de 50000 habitants. Ce n'est qu'en 1940 que São Paulo est devenu l'État le plus peuplé, grâce aux migrations (internes, car le flux des migrations internationales était alors beaucoup plus faible qu'à l'époque de la formation de ses plantations de café), et la croissance rapide du Sudeste a progressivement supplanté le Nordeste en tant que principale région du pays 
Figure 9. Carte de la croissance de la population de 1872 à 2010

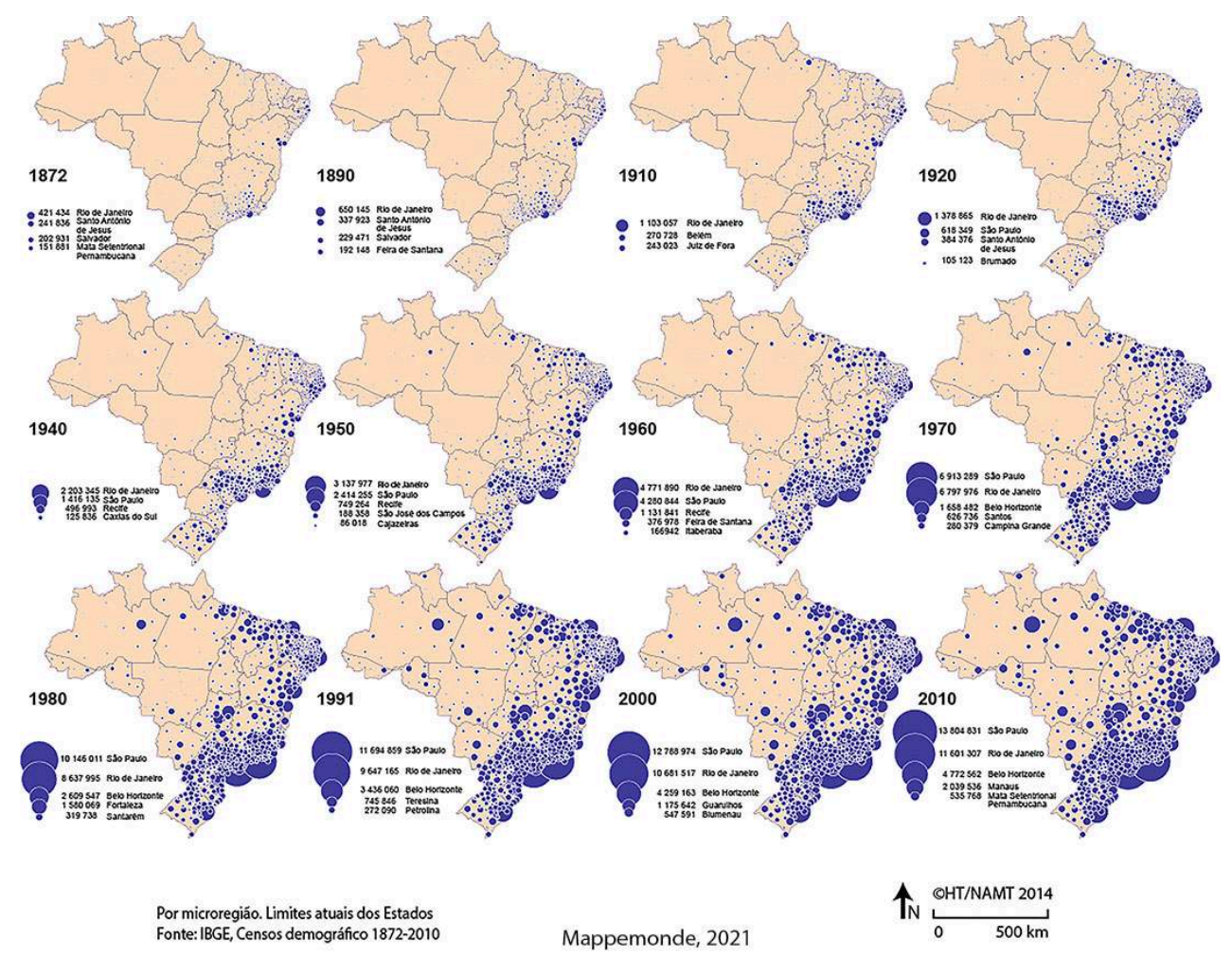

\section{Situation en 2020}

Il pourrait paraître frustrant d'arrêter ces analyses à la date du dernier recensement, en 2010, et de devoir attendre les résultats de celui qui devrait avoir lieu, en principe, en 2021 (il aurait dû être fait en 2020, mais il a été reporté à cause de la pandémie de Covid-19). Toutefois, on dispose heureusement d'une estimation annuelle de la population, toujours grâce à l'IBGE.

Celui-ci a publié le 27 août 2020 l'estimation de la population résidant dans les 5570 communes brésiliennes, selon laquelle le Brésil comptait, à la date de référence du 1er juillet 2019, 211755692 habitants. Ces estimations de la population communale sont obligatoires ${ }^{7}$, car elles sont l'un des paramètres utilisés par la Cour Fédérale des Comptes pour le calcul du Fonds de Participation des États et des Communes et constituent une référence pour divers indicateurs sociaux, économiques et démographiques.

Les notes techniques qui accompagnent la publication de ces estimations annuelles précisent :

«Les estimations démographiques publiées annuellement sont calculées en utilisant [une] méthode mathématique [qui] utilise les populations obtenues à partir des projections démographiques pour le Brésil et les Unités fédérales ${ }^{8}$ les plus récentes comme données de base, ainsi que la croissance démographique de chaque commune dans la dernière décennie, fondée sur les populations respectives enregistrées lors des deux derniers recensements démographiques effectués ».

Sur la carte construite à partir de ces données (figure 10), on constate que la population brésilienne reste très inégalement répartie sur le territoire et qu'il y a toujours une opposition claire entre les régions côtière et intérieure, la première 
densément peuplée et où sont situées presque toutes les grandes villes, et la seconde à faible densité. Même les zones de concentration sont irrégulières, de grands vides apparaissent dans des États densément peuplés. Seuls São Paulo, le Paraná, Rio de Janeiro, Sergipe, Alagoas, Paraíba et Ceará ont des territoires occupés de manière relativement équilibrée.

Dans le reste du territoire, il existe une étroite corrélation entre la répartition de la population et les réseaux de transport, que ce soit avec les voies navigables (en Amazonie), avec les anciennes voies ferrées construites pour exporter des produits de l'intérieur, ou avec les routes qui les ont remplacées.

Figure 10. Population communale du Brésil en 2020

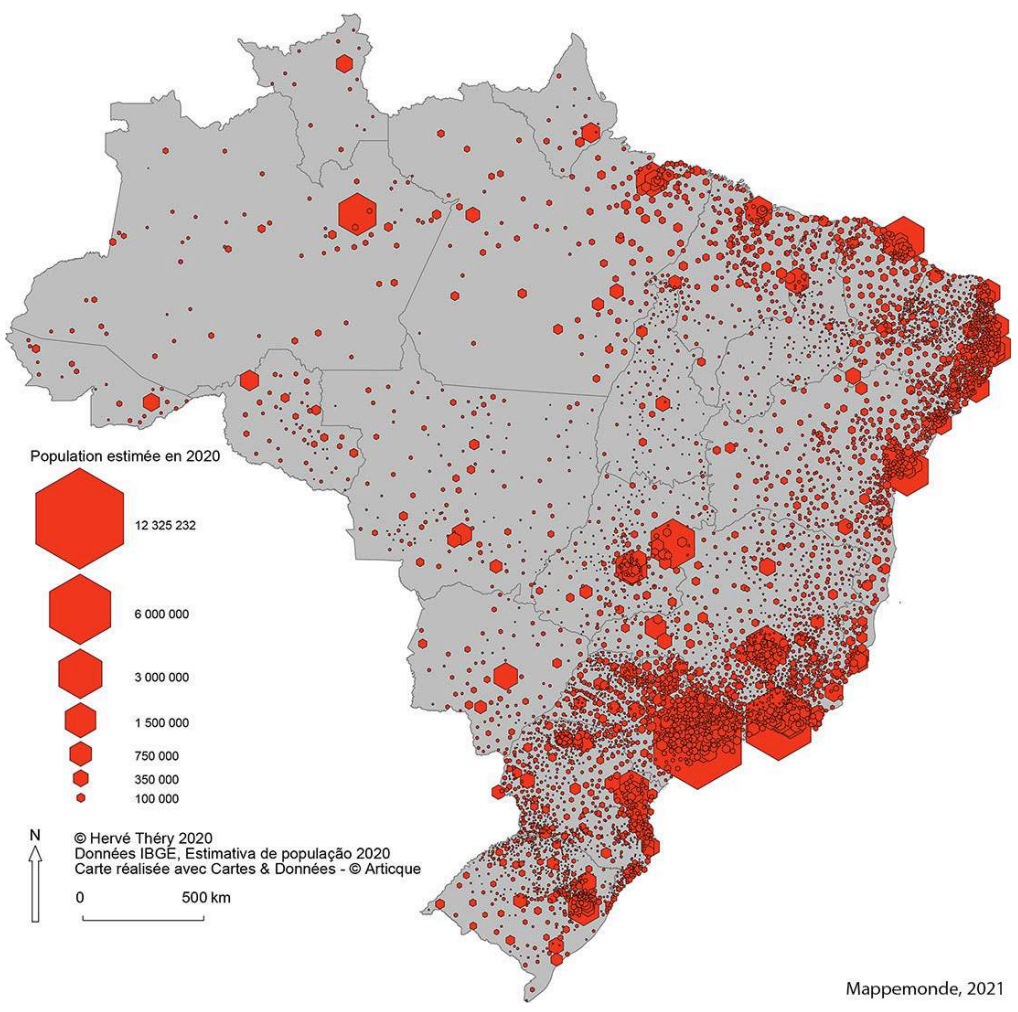

Si l'on utilise les évaluations annuelles de population pour mesurer l'évolution qui s'est produite depuis le dernier recensement (en prenant pour point de départ de la première d'entre elles, en 2011, l'année qui a suivi le recensement), on constate que l'immense majorité des communes brésiliennes voient encore leur population augmenter (figure 11). Il faut, toutefois, distinguer celles où elle augmente modérément, entre 0 et $10 \%$ (ce qui est le cas de la plupart des grandes villes littorales) ou moyennement (entre 10 et $15 \%$ ), et celles où la croissance est forte (de 15 à $75 \%$ ), principalement dans les régions pionnières du centre Ouest et d'Amazonie.

Seul un petit nombre d'entre elles l'ont vu diminuer, dans des régions en difficulté comme le sud de l'État de Bahia, où les plantations de cacao ont été atteintes par la maladie du " balai de sorcière ", le centre Nord du Paraná, le centre du Rondônia, où les pâturages extensifs remplacent progressivement l'agriculture familiale installée lors de la colonisation agraire voulue par l'État dans les années 1970. 
Figure 11. Population, densité démographique et évolution de la population communale du Brésil entre 2011 et 2020

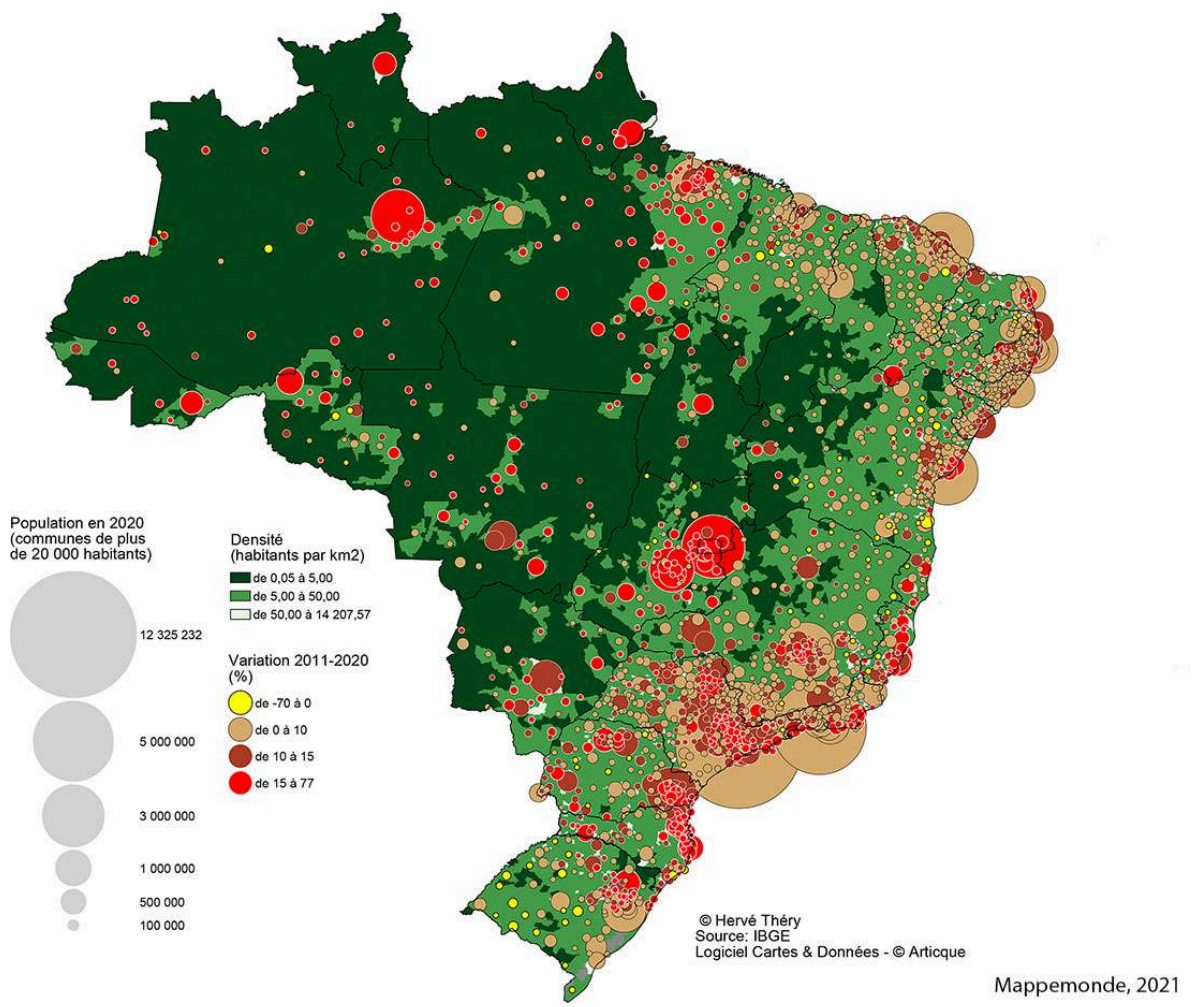

Les données et mailles numériques mises à la disposition des chercheurs, et plus largement du public, par l'IBGE', librement accessibles et gratuites, permettent donc de suivre avec précision l'évolution démographique en remontant dans le temps sur près d'un siècle et demi et pratiquement en temps réel jusqu'à l'année en cours. On ne saurait trop l'encourager à poursuivre cette politique et souhaiter que le recensement de 2020/2021 puisse être réalisé, malgré les difficultés sanitaires et budgétaires du moment, pour fournir des informations fiables sur les évolutions socio démographiques.

\section{NOTES}

1. Chiffre à comparer avec les 35000 communes françaises, alors que le Brésil, avec 8,5 millions de kilomètres carré, est quinze fois plus grand qu'elle.

2. Nos collègues géographes s'en plaignent régulièrement, sans succès pour le moment.

3. Voir, par exemple, " 10 raras fotografias de escravos brasileiros feitas 150 anos atrás " (10 photographies rares d'esclaves brésiliens prises il y a 150 ans), Acervo Instituto Moreira Salles, https://www.historiailustrada.com.br/2014/04/raras-fotografias-escravos-brasileiros.html

4. Voir "Marc Ferrez, un photographe témoin de son temps", Braises, d'où ces photographies sont extraites, et où elles sont référencées. https://braises.hypotheses.org/1602

5. Qui regroupent en ensembles cohérents les 5570 communes actuelles. 
6. «État», car sous la République, proclamée l'année précédente, les anciennes Provinces impériales avaient été ainsi nommées.

7. Cette divulgation annuelle est prescrite par l'article 102 de la loi $n{ }^{\circ} 8$ 443/1992 et la loi complémentaire $n^{\circ} 143 / 2013$.

8. Les États de la Fédération.

9. Certaines sont inattendues, comme le montre le billet «L'Antiquité survit dans les prénoms brésiliens ", Braises, https://braises.hypotheses.org/1140). Il présente une recherche publiée en avril 2016 par l'IBGE, Nomes no Brasil, qui recense les prénoms de tous les résidents du pays, collectés lors dernier recensement démographique, en 2010. On y constate que les porteurs de prénoms antiques, grecs et latins (Heródoto, Temístocles, Diógenes, Deocleciano, Julio Cesar, etc.), sont presque 9,5 millions dans tout le pays. Manifestement l'héritage de l'Antiquité survit au Brésil, transmis avec la langue latine qu'est le portugais.

\section{AUTEUR}

\section{HERVÉ THÉRY}

Directeur de recherche émérite au CNRS-Creda/Professor de posgraduação na Universidade de São Paulo (USP-PPGH) 